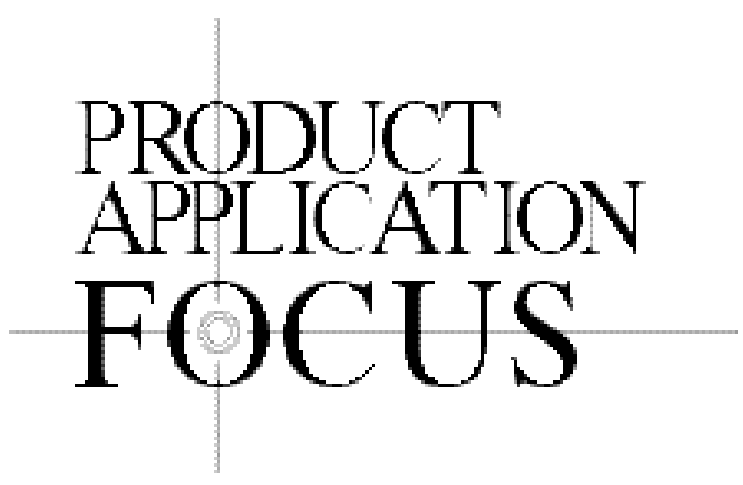

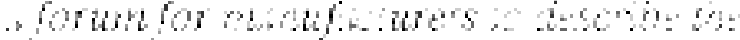

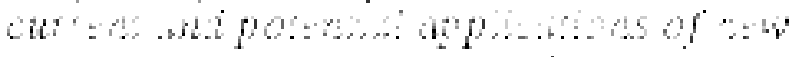

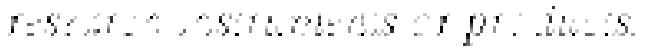

\title{
Optimized T7 Amplification System for Microarray Analysis
}

\author{
C. Pabón, Z. Modrusan, M.V. Ruvolo, I.M. Coleman, S. Daniel, H. Yue, \\ L.J. Arnold, Jr., and M.A. Reynolds \\ Incyte Genomics, Palo Alto, CA, USA
}

BioTechniques 31:874-879(October 2001)

\section{ABSTRACT}

Glass cDNA microarray technologies offer a highly parallel approach for profiling expressed gene sequences in disease-relevant tissues. However, standard hybridization and detection protocols are insufficient for milligram quantities of tissue, such as those derived from needle biopsies. Amplification systems utilizing T7 RNA polymerase can provide multiple cRNA copies from mRNA transcripts, permitting microarray studies with reduced sample inputs. Here, we describe an optimized T7-based amplification system for microarray analysis that yields between 200- and 700-fold amplification. This system was evaluated with both mRNA and total RNA samples and provided microarray sensitivity and precision that are comparable to our standard production process without amplification. The size distributions of amplified cRNA ranged from $200 \mathrm{bp}$ to $4 \mathrm{~kb}$ and were similar to original mRNA profiles. These amplified cRNA samples were fluorescently labeled by reverse transcription and hybridized to microarrays comprising approximately $10000 \mathrm{cDNA}$ targets using a dual-channel format. Replicate hybridization experiments were conducted with the same and different tissues in each channel to assess the sensitivity and precision of differential expression ratios. Statistical analysis of differential expression ratios showed the lower limit of detection to be about 2-fold within and between amplified data sets, and about 3-fold when comparing amplified data to unamplified data (99.5\% confidence).

\section{INTRODUCTION}

Microarrays are rapidly gaining acceptance for gene expression profiling $(4,8,13)$. In particular, glass cDNA microarrays permit the analysis of thousands of gene transcript sequences in a single hybridization experiment (2). Such studies have been used, for example, to associate genes that are expressed differentially between diseased and normal tissues (1). Recently, compendiums of microarray expression data have been described for associating genes within biochemical pathways and deriving new gene targets for therapeutic intervention $(6,12,14)$.

Over the past several years, we have optimized and expanded a glass cDNA microarray (LifeArray ${ }^{\mathrm{TM}}$ ) system for high-throughput gene expression analysis. The performance and attributes of this system were recently described (5). In our standard dual-channel process, Poly $A^{+}$-selected mRNA samples (Poly $\mathrm{A}^{+}$RNA) from two different tissues are labeled with cyanine- 3 and cyanine-5 dyes and hybridized on the microarray. Validation studies have demonstrated that this system can reliably detect about 1:100000 gene transcript sequences starting from $200 \mathrm{ng}$ PolyA $^{+}$RNA sample and provides accurate and precise differential expression ratios over a 2.5-3 $\log _{10}$ dynamic range (16).

A common hurdle for microarray studies is the ability to 
generate RNA samples of sufficient quality and quantity. This is particularly true of needle biopsies and laser capture microdissections, where it is not currently possible to conduct microarray experiments without RNA amplification technologies. Preferably, replicate experiments should be conducted to assign statistical confidence to the differentially expressed genes detected (7). Therefore, robust RNA amplification procedures are needed to advance the capabilities of microarray technology. In 1992, a linear RNA amplification procedure based on T7 RNA polymerase was described for gene expression analysis in single cells (3). Since that time, several investigators have described adaptations of this procedure for microarray hybridization studies $(9,10,15)$. Although the results reported to date are encouraging, questions remain as to the precision and accuracy of this approach. In particular, any skewing of an mRNA population resulting from amplification is expected to be compounded by multiple amplifications, as are commonly employed for very small samples such as laser capture microdissections.

Here, we describe an optimized T7-based system that yields sufficient amplified cRNA for microarray experiments, starting from nanogram quantities of Poly $A^{+}$RNA, or corresponding amounts of unselected total RNA. We also report statistical analysis of replicate hybridization experiments with amplified cRNA samples using glass cDNA microarrays comprising approximately 10000 distinct genes. Our results demonstrate that T7 amplification can be used successfully with as little as $1 \mathrm{ng}$ input Poly $\mathrm{A}^{+} \mathrm{RNA}$, or a corresponding amount of total RNA, and provide microarray expression data of similar quality compared to our standard production process without amplification.

\section{MATERIALS AND METHODS}

\section{RNA Isolation and Quantitation}

Human diseased kidney tissues (matched tumor and normal biopsies) were weighed and then homogenized in TRIzol ${ }^{\circledR}$ buffer (Invitrogen, Carlsbad, CA, USA) at the Incyte Genomics Core RNA Facility (Palo Alto, CA, USA). A dilution series of each lysate was prepared in TRIzol buffer, and individual sam ples of total RNA were then precipitated with the addition of isopropanol and glycogen (Ambion, Austin, TX, USA). Human total RNA from normal tissues (brain and placenta) was purchased from the BioChain Institute (Hayward, CA, USA). Poly $A^{+}$RNA was prepared from total RNA samples using an Oligotex ${ }^{\circledR}$ mRNA isolation kit (Qiagen, Valencia, CA, USA). The resulting total RNA and mRNA samples were quantitated by fluorescence spectroscopy using a RiboGreen ${ }^{\circledR}$ RNA Quantitation Assay (Molecular Probes, Eugene, OR, USA) with modifications described previously (16). Averaged fluorescence values from triplicate measurements were compared to a seven-step standard curve to determine RNA concentrations.

\section{T7 RNA Amplification}

RNA samples were subjected to a linear amplification using a T7-based amplification kit (Incyte Genomics). The resulting amplified cRNA samples were quantitated as described in the previous section. Relative values for RNA amplification were calculated as (mass amplified cRNA)/(mass input mRNA).

\section{Transcript Profiling}

The size distributions of input Poly $\mathrm{A}^{+}$RNA samples and amplified cRNA products were determined using a high-resolution electrophoresis system, the Agilent 2100 Bioanalyzer $^{\mathrm{TM}}$ (Agilent, Palo Alto, CA, USA). This instrument separates RNA fragments through microfabricated channels with real-time fluorescence detection. RNA samples were first diluted with injection buffer according to the manufacturer's protocol and then analyzed in parallel with an external RNA 6000 size ladder (Ambion).

\section{Probe Generation and Hybridization on Glass cDNA Microarrays}

Amplified cRNA and unamplified mRNA samples (200 ng/reaction) were fluorescently labeled by reverse transcription with cyanine- 3 and cyanine- 5 dyes (LifeArray microarray labeling kits; Incyte Genomics) as described previously (16). For dual-channel hybridization experiments, cyanine-3and cyanine-5-labeled cDNA probes were combined and purified by size exclusion chromatography (Chroma Spin ${ }^{\mathrm{TM}}$ TE30 column; Clontech Laboratories, Palo Alto, CA), ethanol precipitated, and then resuspended in $24 \mu \mathrm{L}$ hybridization buffer $(5 \times$ SSC, $0.2 \%$ SDS, 1 mM DTT $)$.

Hybridization experiments were performed on LifeArray Human Drug Target microarrays (Incyte Genomics). These microarrays comprise 9738 sequence-verified human cDNAs representing 8443 unique annotated EST clusters. For each comparison of conditions, the replicates were run in parallel using input RNA samples from the same tissue sources. The fluorescently labeled hybridization probes were applied to the microarrays under $22 \times 22-\mathrm{mm}$ glass cover slips, placed in sealed chambers to prevent evaporation, and then incubated at $60^{\circ} \mathrm{C}$ for $6 \mathrm{~h}$. After hybridization, the microarrays were washed in $1 \times \mathrm{SSC} / 0.1 \% \mathrm{SDS}$ at $45^{\circ} \mathrm{C}$ for $10 \mathrm{~min}$ and then in $0.1 \times \mathrm{SSC} / 0.2 \% \mathrm{SDS}$ at $25^{\circ} \mathrm{C}$ for $3 \mathrm{~min}$.

\section{Scanning and Data Analysis}

The microarrays were scanned with excitation at $532 \mathrm{~nm}$ (cyanine-3) and $635 \mathrm{~nm}$ (cyanine-5) using a GenePix ${ }^{\mathrm{TM}}$ 4000B (Axon Instruments, Foster City, CA, USA) at $10 \mu \mathrm{m}$ resolution. The fluorescent signal was collected with 16bit/pixel resolution. GEMTools ${ }^{\mathrm{TM}}$ image analysis software (Incyte Genomics) was used to derive corrected signal intensities at each element with a signal-to-background threshold of 2.5. Balanced differential expression ratios were calculated from the corrected signal intensities at each element. The measured differential expression ratios were converted to natural log space and then averaged for graphical comparisons and statistical analysis. Data manipulations and analysis were conducted using GEMTools ${ }^{\mathrm{TM}}$, Microsoft ${ }^{\circledR}$ Access ${ }^{\circledR}$, and Statistica ${ }^{\circledR}$ software (StatSoft, Tulsa, OK, USA). Limits of detection were calculated at a $99.5 \%$ confidence and are expressed in units of differential expression (16). 


\section{RESULTS AND DISCUSSION}

Commonly used labeling procedures for microarray experiments require $50-200 \mu \mathrm{g}$ total RNA or $2-5 \mu \mathrm{g}$ Poly A ${ }^{+}$RNA (2). Reported microarray studies employing T7-based amplification have specified a minimum of $2.5-3 \mu \mathrm{g}$ amplified RNA per hybridization for optimal results $(10,11,15)$. Based on this requirement, many investigators have resorted to two or more rounds of $\mathrm{T} 7$ amplification to obtain sufficient amounts of amplified RNA for microarray experiments $(9,11,15)$. However, because the LifeArray probe labeling system requires considerably less RNA sample (200 ng Poly $A^{+}$RNA per channel), we recognized that a single round of T7 amplification might be sufficient for many "low-input" samples. Therefore, the Incyte T7 amplification system was optimized as a single-round cRNA synthesis and validated with mRNA and total RNA samples as described below.

\section{Microarray Performance with Amplified mRNA}

The T7 amplification system was first investigated with Poly A+ RNA samples (1- and 10-ng inputs) because unamplified Poly $\mathrm{A}^{+} \mathrm{RNA}$ is typically used in our standard production process. This study was conducted to provide a direct comparison between amplified and unamplified PolyA $\mathrm{A}^{+} \mathrm{RNA}$ samples from the same tissue sources. As summarized in Table 1, the T7 system provided about 700-fold amplification, making it possible to run multiple microarray experiments with as little as $1 \mathrm{ng}$ input Poly $\mathrm{A}^{+}$RNA. As shown in Figure 1 , the size distributions of amplified cRNAs were similar for both 10- and 1-ng inputs, with the majority ranging between 200 bases and $4 \mathrm{~kb}$ in length.

The first set of experiments was conducted with amplified cRNAs from the same tissue source in both channels to determine the lower limit of detection (LLOD) that could be achieved based on statistical variance (16). Theoretically, there should be no difference in balanced signal values between the two channels, and all of the resulting data should show a balanced differential expression ratio of 1 . As shown in Figure 2, microarray hybridization results were highly comparable between 10- and 1-ng inputs, showing a similar dynamic range and distribution of data points along the sym metry axis. Scatter around the symmetry axis was similar across the entire dynamic range, indicating a negligible loss

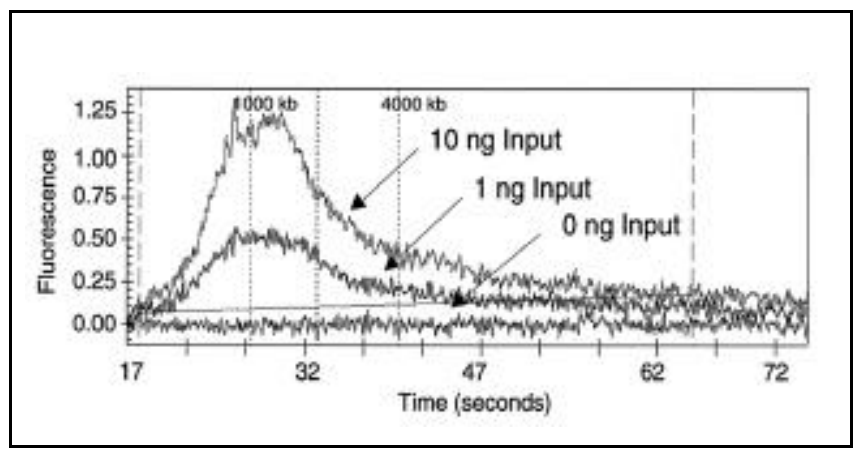

Figure 1. Microcapillary electrophoretic profiles for amplified cRNA products derived from 1- and 10-ng Poly $\mathrm{A}^{+} \mathrm{RNA}$ inputs or no input.
Table 1. Summary of Averaged cRNA Yields $(n=5)$ for Poly $A^{+}$-Selected RNA Inputs

\begin{tabular}{|lccc|}
\hline $\begin{array}{l}\text { Input Poly } A^{+} \\
\text {RNA (ng) }\end{array}$ & Tissue & $\begin{array}{c}\text { Average Yield } \\
\text { cRNA (ng) }\end{array}$ & $\begin{array}{c}\text { Fold } \\
\text { Amplification }\end{array}$ \\
\hline 10 & Placenta & 7380 & 738 \\
10 & Brain & 7840 & 784 \\
1 & Placenta & 698 & 698 \\
1 & Brain & 752 & 752 \\
\hline
\end{tabular}

of precision as the signals approach background. The coefficients of variance from replicate microarray experiments $(n=$ 5 for each input) for 10 and $1 \mathrm{ng}$ Poly $\mathrm{A}^{+}$RNA inputs were $19.3 \%$ and $19.7 \%$, respectively. Based on these values, differential expression ratios greater than or equal to 2-fold can be measured with greater than $99.5 \%$ statistical confidence.

Microarray hybridization experiments were next conducted with amplified and unamplified PolyA ${ }^{+}$RNA from two different tissues to provide a direct comparison of differential expression ratios. The numbers of ratio values returned from replicate hybridizations are summarized in Table 2 . The percentage of ratio values returned from individual hybridiza-

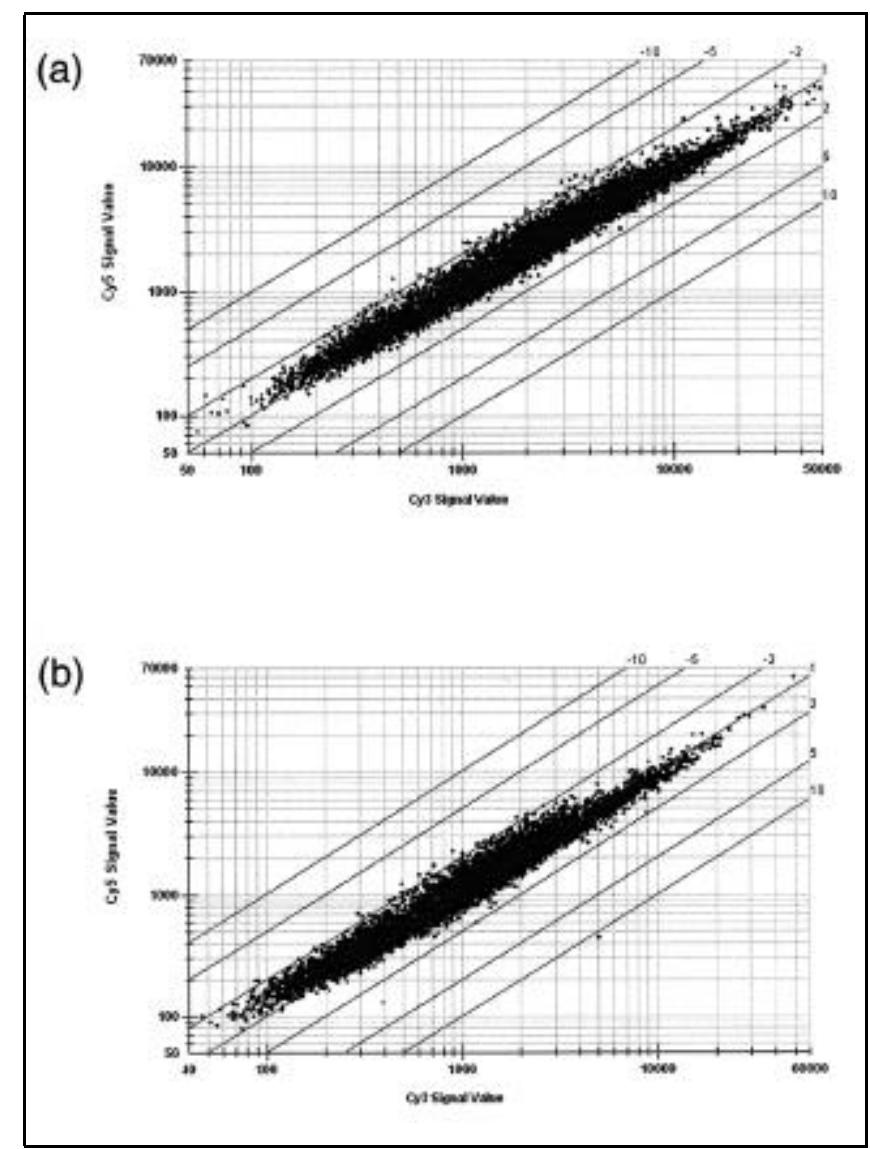

Figure 2. Representative scatter plots from dual-channel microarray hybridizations with amplified cRNA samples from the same tissue source in both channels. (a) Amplified cRNA generated from $10 \mathrm{ng}$ input Poly $\mathrm{A}^{+}$ RNA. (b) Amplified cRNA generated from 1 ng input PolyA RNA. 
Table 2. Positive Elements Returned from Dual Tissue Microarray Hybridizations (9738 Total Elements)

\begin{tabular}{|lllll|}
\hline Microarray & $\begin{array}{c}\text { Tissues } \\
\text { (Cy3/Cy5) }\end{array}$ & $\begin{array}{c}\text { RNA Type } \\
\text { (ng input) }\end{array}$ & $\begin{array}{c}\text { No. Ratio } \\
\text { Values }\end{array}$ & $\begin{array}{c}\text { No. Common } \\
\text { Ratio Values }\end{array}$ \\
\hline 1 & Placenta/Brain & cRNA (10) & 9387 & 9086 \\
2 & Placenta/Brain & cRNA (10) & 9616 & 9603 \\
4 & Placenta/Brain & cRNA (10) & 9320 \\
5 & Placenta/Brain & cRNA (10) & 9443 \\
\hline 6 & Placenta/Brain & cRNA (10) & 9802 \\
7 & Placenta/Brain & cRNA (1) & 9142 \\
10 & Placenta/Brain & cRNA (1) & 9607 \\
\hline 11 & Placenta/Brain & cRNA (1) & 9292 \\
12 & Placenta/Brain & cRNA (1) & 9034 \\
\hline 13 & Placenta/Brain & cRNA (1) & 8788 \\
\hline 16 & Brain/Placenta & cRNA (10) & 8818 \\
\hline
\end{tabular}

tions ranged from $88 \%$ to $98 \%$ (versus the total number of 9738 elements present on the array). In addition, the percentage of ratio values that were commonly returned from each set of replicates ranged from $89 \%$ to $93 \%$. These high percentages permitted averaged ratio values to be used for com parisons with the vast majority of the data accounted for.

As shown in Figure 3, the averaged differential expression ratios derived from amplified cRNA were highly comparable between the 10- and 1-ng inputs (8667 common ratio values,

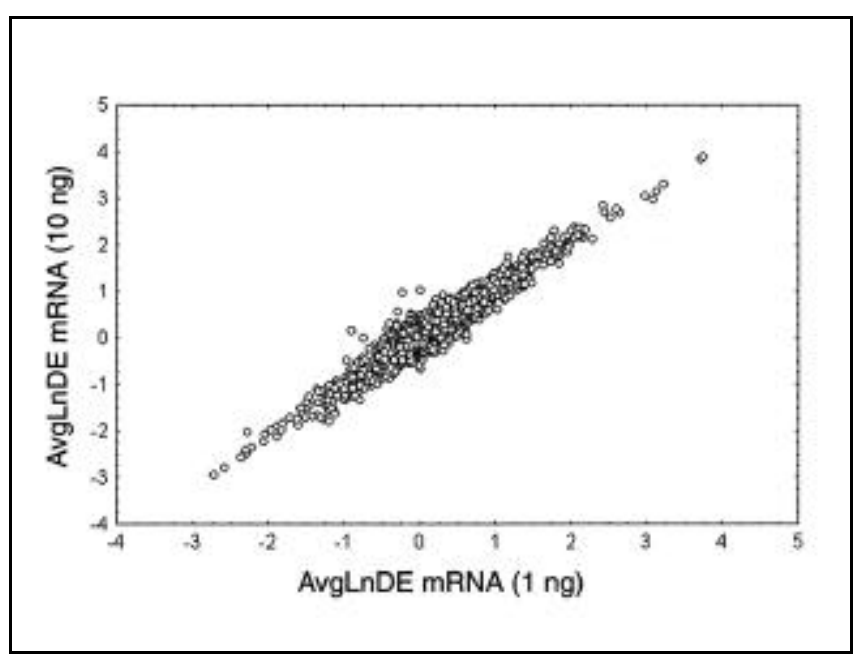

Figure 3. Scatter plot comparison of averaged differential expression ratios generated from amplified Poly $\mathrm{A}^{+} \mathrm{RNA}$ at two different inputs ( 1 and $10 \mathrm{ng}$, respectively). Each set of microarray hybridizations was conducted in a dual-channel format (see Materials and Methods) using input Poly $\$$ RNA from the same tissue sources (Cy3 = human brain; $\mathrm{Cy} 5=$ human placenta). slope $=0.906)$. The coefficient of variance between these two data sets was $14.2 \%$. Using a differential expression ratio cutoff of 2-fold, there were no discordant ratios between these two data sets. A similar comparison was made between amplified cRNA (10-ng inputs) and unamplified mRNA (8628 common ratio values). In this case, the coefficient of variance was $29.9 \%$, indicating that a threshold of about 3 -fold should be used when comparing ratios between data sets.

\section{Microarray Performance with Amplified Total RNA}

Aliquots of total RNA derived from human brain and placenta tissue were subjected to Poly $A^{+}$selection to provide samples of both total and PolyA $A^{+}$RNA from the same tissue sources (see Materials and Methods). The optimized T7 am plification system was then applied to these total and Poly $\mathrm{A}^{+}$ RNA samples ( $1 \mu \mathrm{g}$ and $10 \mathrm{ng}$, respectively). Representative size distribution profiles for amplified cRNA products are shown in Figure 4a. In most cases, cRNAs derived from total RNA showed slightly longer and broader size distributions compared to those derived from Poly $A^{+}$RNA.

Replicate microarray hybridizations were conducted with amplified cRNAs derived from total and PolyA+ RNA samples, respectively. As shown in Figure 4b, averaged differential expression ratios derived from total RNA inputs were substantially similar to those derived from Poly $A^{+}$RNA inputs (8603 common ratio values, slope $=1.05)$. Again, there were no discordant ratios between the two types of RNA inputs using a differential expression ratio threshold of 2.0. This demonstrates that amplified cRNAs derived from total RNA and mRNA inputs give statistically similar results in microarray experiments. 


\section{T7 RNA Amplification from Tissue Lysates}

This study was conducted to demonstrate that the T7 am plification system could be applied directly to tissue lysates, in addition to dilutions of purified RNA as described above. Lysates of human kidney tissue (matched normal and tumor biopsies) were aliquoted as a dilution series corresponding to a range of 1.7-16.7 mg tissue/sample. Each sample was subjected individually to total RNA isolation. As shown in Figure $5 \mathrm{a}$, the size distributions of amplified cRNA products were similar throughout the range of inputs. Furthermore, consistent yields of amplified cRNA were observed for all inputs (Figure 5b). Amplified cRNA products corresponding to a specified amount of normal or tumor tissue were then subjected to microarray analysis. Statistical analysis of differential expression ratios resulting from replicate microarray hybridizations is summarized in Table 3 . A modest reduction in the number of reported ratio values was observed as inputs of tissue lysate were decreased from $16.7 \mathrm{mg}$ (about 250000 cells) to $1.7 \mathrm{mg}$ (about 25000 cells). In addition, there was a slight increase in the coefficient of variance within replicates. Taken together, these results demonstrate a practical lower limit for our T7 amplification system on the order of 1-5 mg tissue. It should be pointed out that these results were generated from a single matched set of tissue biopsies. Actual results may be more or less favorable than those reported here,

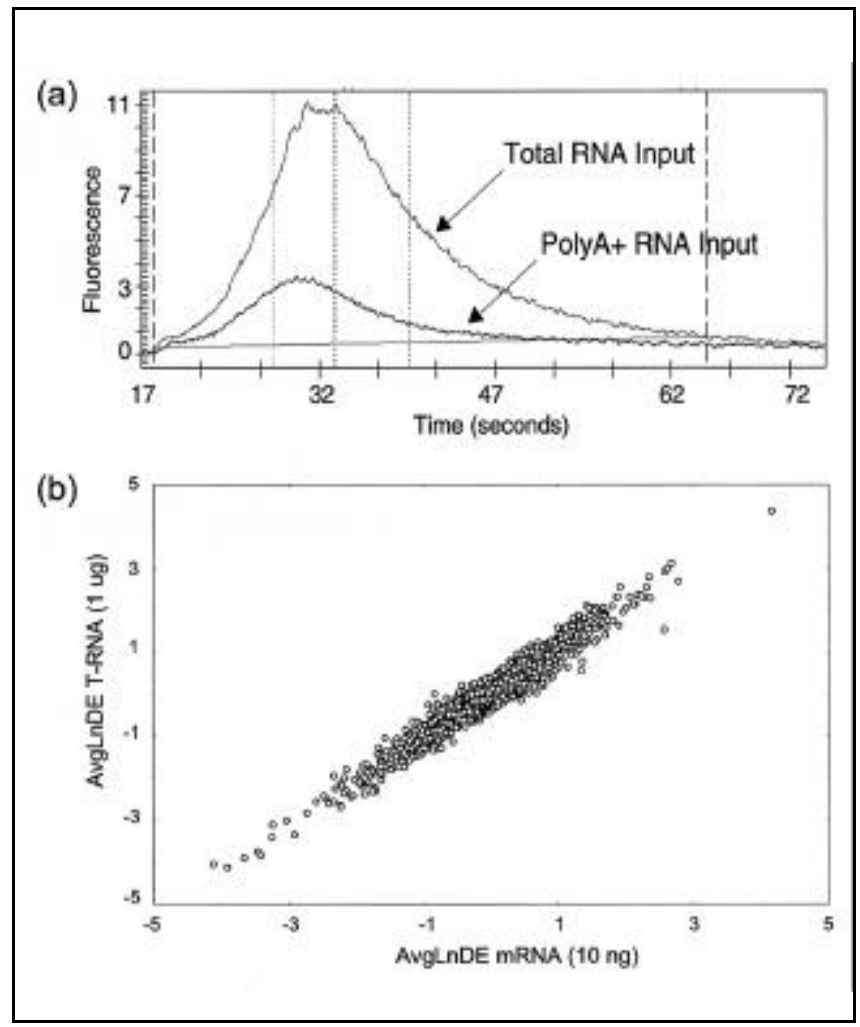

Figure 4. Comparison of $\mathrm{T} 7$ amplification results using total and Poly $\mathrm{A}^{+}$ RNA inputs from the same tissue sources. (a) Microcapillary electrophoretic profiles for amplified cRNA from PolyA + RNA (10 ng) and total RNA $(1 \mu \mathrm{g})$ inputs. (b) Scatter plot comparison of averaged differential expression ratios derived from amplified mRNA and total RNA.
Table 3. Microarray Hybridization Results with T7-Amplified cRNAs Derived from Human Kidney Lysates (Matched Normal and Tumor Biopsies)

\begin{tabular}{|ccc|}
\hline $\begin{array}{c}\text { Tissue Inputs } \\
\text { (mg) }\end{array}$ & $\begin{array}{c}\text { No. Common } \\
\text { Expression Ratios }\end{array}$ & $\begin{array}{c}\text { \%cv } \\
\text { (Ratio Values) }\end{array}$ \\
\hline 16.7 & 8152 & 9.8 \\
5.0 & 7979 & 10.1 \\
3.3 & 7969 & 11.6 \\
1.7 & 7527 & 13.7 \\
Three independent microarray hybridizations were run for \\
each condition.
\end{tabular}

depending on the condition of the tissue being investigated (processing, cryostorage, etc.).

\section{CONCLUSION}

Based on the data presented, our optimized T7 amplification system is capable of generating reliable differential expression ratios using nanogram quantities of Poly $A^{+}$RNA. Microarray expression data derived from amplified cRNA showed similar precision to our standard production process

(a)

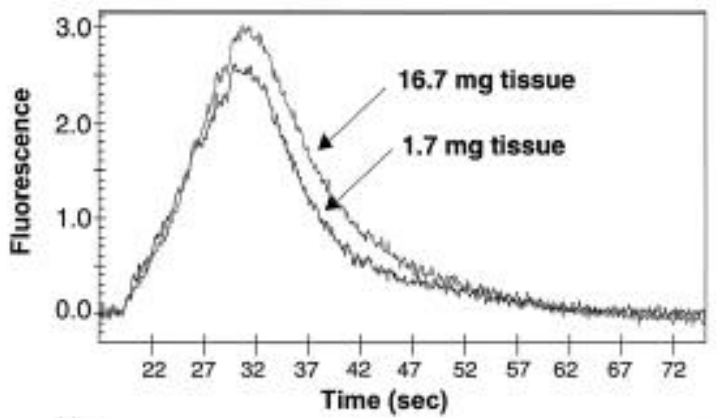

(b)

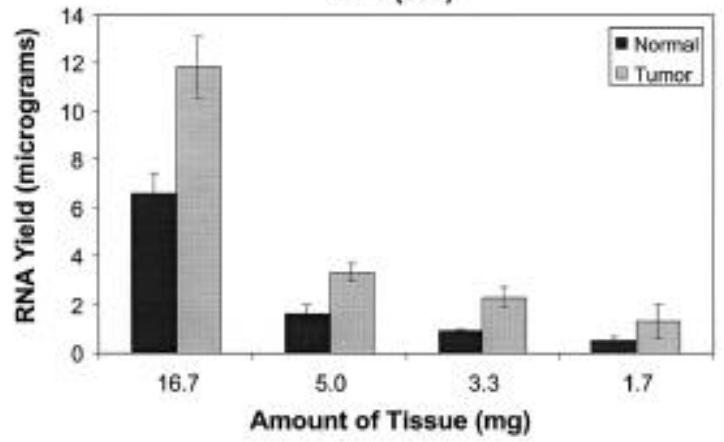

Figure 5. T7 amplification results using input total RNA derived from milligram-scale tissue lysates. (a) Microcapillary electrophoretic profiles for amplified cRNA derived from 1.7 and $16.7 \mathrm{mg}$ tumor tissue lysates. (Sim ilar profiles were obtained from the matched normal tissue lysates.) (b) cRNA quantitation results from replicate $\mathrm{T} 7$ amplification reactions over a range from 16.7 to $1.7 \mathrm{mg}$ tissue lysate. 
without amplification (16). Based on the data presented, we conclude that the T7 amplification system can provide accurate differential expression ratios greater than or equal to 2fold at $99.5 \%$ confidence or higher. Comparisons of microarray hybridization data generated from amplified cRNA versus unamplified Poly $\mathrm{A}^{+}$RNA showed a similar number of returned ratio values, with no evidence of signal compression in the former case (i.e., reduced dynamic range). However, our results indicate that a slightly higher expression ratio threshold (about 3-fold) should be applied when comparing ratios derived from cRNA versus PolyA $A^{+}$RNA.

Our T7 amplification system can also be applied directly to total RNA samples without selection. Our comparative hybridization results showed a very good correlation between differential expression ratios derived from total RNA versus Poly $A^{+}$RNA inputs. Furthermore, the system could be applied directly to precipitated RNA from tissue lysates with good reproducibility.

Since completing the study described above, we have completed a number of microarray experiments with $\mathrm{T} 7$ amplified RNA samples from disease-relevant tissues. In practice, we have routinely obtained 200- to 500-fold amplification, slightly lower than reported here. These variations could be explained by differences in the quality of the input PolyA ${ }^{+}$RNA samples.
11.Ohyama, H., X. Zhang, Y. Kohno, I. Alevisos, M. Posner, D.T. Wong, and R. Todd. 2000. Laser capture microdissection-generated target sam ple for high-density oligonucleotide array hybridization. BioTechniques 29:530-536.

12.Perou, C.M., T. Sorlie, M.B. Eisen, M. van de Rijn, S.S. Jeffrey, C.A. Rees, J.R. Pollack, D.T. Ross et al. 2000. Molecular portraits of human breast tumours. Nature 406:747-752.

13.Ramsay, G. 1998. DNA chips: state-of-the art. Nat. Biotechnol. 16:40-44.

14.Scherf, U., D.T. Ross, M. Waltham, L.H. Smith, J.K. Lee, L. Tanabe, K.W. Kohn, W.C. Reinhold et al. 2000. A gene expression database for the molecular pharmacology of cancer. Nat. Genet. 24:236-244.

15.Wang, E., L.D. Miller, G.A. Ohnmacht, E.T. Liu, and F.M. Marincola. 2000. High-fidelity mRNA amplification for gene profiling. Nat. Biotechnol. 18:457-459.

16. Yue, H., P.S. Eastman, B.B. Wang, J. Minor, M.H. Doctolero, R.L. Nuttall, R. Stack, J.W. Becker et al. 2001. An evaluation of the performance of cDNA microarrays for detecting changes in global mRNA expression. Nucleic Acids Res. 29:e41-e50.

Address correspondence to Dr. Mark A. Reynolds, Incyte Genomics, 3160 Porter Avenue, Palo Alto, CA 94304, USA. e-mail:markr@incyte.com

For reprints of this or any other article, contact Reprints@BioTechniques.com

\section{ACKNOWLEDGMENTS}

We thank Bruce Wang and Gordon Porter for early contributions to the RNA amplification protocols described in this study. We also thank Scott Eastman and Tom Theriault for technical guidance and for critically reviewing this manuscript.

\section{REFERENCES}

1.DeRisi, J., L. Penland, P.O. Brown, M.L. Bittner, P.S. Meltzer, M. Ray, Y. Chen, Y.A. Su, and J.M. Trent. 1996. Use of a cDNA microarray to analyse gene expression patterns in human cancer. Nat. Genet. 14:457-460.

2.Duggan, D.J., M. Bittner, Y. Chen, P. Meltzer, and J.M. Trent. 1999. Expression profiling using cDNA microarrays. Nat. Genet. 21:10-14.

3.Eberwine, J., H. Yeh, K. Miyashiro, Y. Cao, S. Nair, R. Finnell, M. Zettel, and P. Coleman. 1992. Analysis of gene expression in single live neurons. Proc. Natl. Acad. Sci. USA 89:3010-3014.

4.Epstein, C.B. and R.A. Butow. 2000. Microarray technology-enhanced versatility, persistent challenge. Curr. Opin. Biotechnol. 11:36-41.

5.Evertsz, E., P. Starink, R. Gupta, and D. Watson. 2000. Technology and applications of gene expression microarrays, p. 149-166. In M. Schena (Ed.), Microarray Biochip Technology. BioTechniques Books Division, Natick, MA.

6.Hughes, T.R., C.J. Roberts, H. Dai, A.R. Jones, M.R. Meyer, D. Slade, J. Burchard, S. Dow et al. 2000. Widespread aneuploidy revealed by DNA microarray expression profiling. Nat. Genet. 25:333-337.

7.Lee, M.L., F.C. Kuo, G.A. Whitmore and J. Sklar. 2000. Importance of replication in microarray gene expression studies: statistical methods and evidence from repetitive cDNA hybridizations. Proc. Natl. Acad. Sci. USA 97:9834-9839.

8.Lennon, G.G. 2000. High-throughput gene expression analysis for drug discovery. Drug. Discov. Today 5:59-66.

9.Luo, L., R.C. Salunga, H. Guo, A. Bittner, K.C. Joy, J.E. Galindo, X. Xiao, K.E. Rogers et al. 1999. Gene expression profiles of laser-captured adjacent neuronal subtypes. Nat. Med. 5:117-122.

10.Mahadevappa, M. and J.A. Warrington. 1999. A high-density probe array sample preparation method using 10- to 100 -fold fewer cells. Nat. Biotechnol. 17:1134-1136. 